\title{
microRNA-520a-3p inhibits proliferation and cancer stem cell phenotype by targeting HOXD8 in non-small cell lung cancer
}

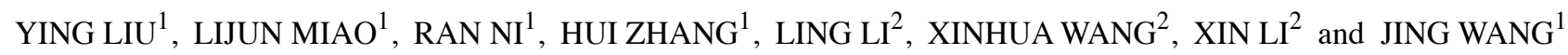 \\ Departments of ${ }^{1}$ Respiratory Medicine and ${ }^{2}$ Oncology Medicine, \\ The First Affiliated Hospital of Zhengzhou University, Zhengzhou, Henan 450052, P.R. China
}

Received April 17, 2016; Accepted August 19, 2016

DOI: $10.3892 /$ or.2016.5149

\begin{abstract}
Formation of cancer stem cells (CSCs) and increased cells proliferation are involved in tumorigenesis, tumour recurrence and therapy resistance and microRNA is essential for the development of the biological traits of CSCs and the increased cells proliferation. Studying molecular mechanism of tumorigenesis, tumour recurrence and therapy resistance of lung cancer will help us to further understand the pathogenesis and progression of the disease and offer new targets for effective therapies. In the present study, we found that miR-520a-3p expression is downregulated in NSCLC (non-small cell lung cancer) and SCLC (small cell lung cancer). miR-520a-3p can inhibit proliferation and cancer stem cell phenotype in NSCLC and SCLC cells. Overexpressing miR-520a-3p can degrade HOXD8 mRNA in NSCLC cells, but its overexpression cannot suppress HOXD8 in SCLC cells. HOXD8 protein is upregulated in NSCLC tissues and its overexpression can promote proliferation, formation of cancer stem cells, migration and invasion in NSCLC cells. MET amplification plays a pivotal role in gefitinib resistance in lung cancer. We found that miR-520a-3p can downregulate MET protein expression and HOXD8 can upregulate MET protein expression. Thus, we concluded that microRNA-520a-3p inhibits proliferation and cancer stem cell phenotype by targeting HOXD8 in NSCLC cells and restoration of microRNA-520a-3p might be a therapeutic strategy to reverse gefitinib resistance.
\end{abstract}

Correspondence to: Dr Jing Wang, Department of Respiratory Medicine, The First Affiliated Hospital of Zhengzhou University, No. 1 Jianshe East Road, Erqi District, Zhengzhou, Henan 450052, P.R. China

E-mail: wangjingzzu@126.com

Abbreviations: ALDH1A1, aldehyde dehydrogenase 1; ANT, adjacent normal tissues; ASCL1, achaete-scute complex homolog 1; CSCs, cancer stem cells; CICs, cancer initiating cells; NSCLC, non-small cell lung cancer; SCLC, small cell lung cancer

Key words: CSCs, proliferation, miR-520a-3p, HOXD8, non-small cell lung cancer; small cell lung cancer

\section{Introduction}

Lung cancer is the leading cause of cancer-related mortality worldwide. Non-small cell lung cancer (NSCLC) accounts for approximately $80 \%$ of lung cancers. Despite considerable progress in diagnosis and treatment, the overall 5-year survival rate of NSCLC patients still remains $<15 \%(1,2)$. These findings underscore that elucidating molecular mechanism of pathogenesis and progression of lung cancer will offer novel targets for effective therapies.

Cancer stem cells (CSCs) or cancer initiating cells (CICs) are a rare subpopulation of undifferentiated cells that are responsible for tumor initiation, maintenance and spreading (3-5). They have been identified in various human malignancies, including breast, brain, prostate, pancreatic, colon and lung cancer (6-11). CSCs have been blamed for playing a critical role in drug resistance and cancer metastasis, which may explain why it is difficult to completely eradicate cancer and why recurrence is a real threat in eradication of tumors completely (12-14).

MicroRNAs (miRNAs/miRs) are small non-coding RNAs of 20-22 nucleotides that have been implicated in various types of cancer (15-17). Abnormal miRNA expression has been linked to diseases, including lung cancer and it has been found implicated in a multitude of cellular processes including proliferation, differentiation, invasion, migration and apoptosis $(16,18-24)$. MicroRNAs (miRNAs) regulate both normal stem cells and CSCs and miRNA dysregulation has been implicated in tumorigenesis (16,25-28). Recently, it has been reported that miR-520a-3p can inhibit proliferation, apoptosis and metastasis in NSCLC by targeting MAP3K2, and miR-520a-3p may be used as a prognostic marker for NSCLC in clinical research (29). However, its roles still keep emerging in lung cancer.

In the present study, we found that miR-520a-3p expression is downregulated in NSCLC (non-small cell lung cancer) and SCLC (small cell lung cancer). miR-520a-3p can inhibit proliferation and cancer stem cell phenotype in NSCLC and SCLC cells. Overexpressing miR-520a-3p can degrade HOXD8 mRNA in NSCLC cells, but its overexpression cannot suppress HOXD8 in SCLC cells. HOXD8 protein is upregulated in NSCLC tissues and its overexpression can promote proliferation, formation of cancer stem cells, migration and invasion in NSCLC cells. MET amplification play a pivotal 
role in gefitinib resistance in lung cancer. We found that miR-520a-3p can downregulate MET protein expression and HOXD8 can upregulate MET protein expression, implying that miR-520a-3p and HOXD8 might be potential targets for the therapy of NSCLC. Thus, we concluded that microRNA520a-3p inhibits proliferation and cancer stem cell phenotype by targeting HOXD8 in NSCLC cells and restoration of microRNA-520a-3p might be a therapeutic strategy to reverse gefitinib resistance.

\section{Materials and methods}

NSCLC and SCLC tissues. Lung cancer tissues and adjacent normal tissues were obtained from the First Affiliated Hospital of Zhengzhou University. All tissues (20 NSCLC tissues and 19 SCLC tissues) were examined histologically, and pathologists confirmed the diagnosis. Medical ethics committee has approved the experiments undertaken. The use of human's tissue samples follows internationally recognized guidelines as well as local and national regulations. Informed consent was obtained from each individual.

NSCLC and SCLC cell lines. NSCLC cell line A549 and SCLC cell line NCI-H1688 were obtained from the American Type Culture Collection (ATCC; Manassas, VA, USA). Cells were grown in culture medium, as recommended by the ATCC. Cells were cultured in Dulbecco's modified Eagle's medium (DMEM; Gibco-Invitrogen, Carlsbad, CA, USA) containing $10 \%$ fetal calf serum (FCS), $2 \mathrm{mM} \mathrm{L-glutamine,} 100 \mathrm{U} / \mathrm{ml}$ penicillin and $100 \mu \mathrm{g} / \mathrm{ml}$ streptomycin at $37^{\circ} \mathrm{C}$ in a humidified $5 \% \mathrm{CO}_{2}$ atmosphere.

Pre-miR-520a-3p/control miR, HOXD8 expressing plasmids/empty vectors and transfection. Pre-miR-520a-3p and control-miR were purchased from Ambion (Austin, TX, USA). A final concentration of $50 \mathrm{nM}$ of Pre-miR-520a-3p and its respective negative control (control-miR) were used for each transfection. HOXD8 expressing plasmids/ empty vectors were purchased from Tiangen Biotech, Co., Ltd. (Beijing, China). A final concentration of $10 \mu \mathrm{g}$ of HOXD8 expressing plasmids and its respective negative control (empty vectors) were used for each transfection. For the transfection experiments, the cells were cultured in serum-free medium without antibiotics at $60 \%$ confluence for $24 \mathrm{~h}$, and then transfected with transfection reagent (Lipofectamine 2000; Invitrogen) according to the manufacturer's instructions. After incubation for $6 \mathrm{~h}$, the medium was removed and was replaced with normal culture medium for $48 \mathrm{~h}$, unless otherwise specified.

Real-time PCR for miRNA. Total RNA from cultured cells, with efficient recovery of small RNAs, was isolated using the mirVana miRNA isolation kit (Ambion). Detection of the mature form of miRNAs was performed using the mirVana qRT-PCR miRNA detection kit, according to the manufacturer's instructions (Ambion). The U6 small nuclear RNA was used as an internal control.

MTT assay. MTT assay was performed as previously described (31).
Sphere growth. Cells $\left(10^{3} / \mathrm{ml}\right)$ in serum-free RPMI- $1640 / 1 \mathrm{mM}$ Na-pyruvate were seeded on $0.5 \%$ agar precoated 6-well plates. After 1 week, half the medium was changed every third day. Single spheres were picked and counted.

Western blot analysis. Western blot analysis was performed as previously described (22). Mainly, after incubation with primary antibody anti-ASCL1 (1:500; Abcam, Cambridge, MA, USA), antibody anti-ALDH1A1 (1:500; Abcam), antiHOXD8 (1:500; Abcam), anti-p53 (1:500; Abcam), anti-PTEN (1:500; Abcam), anti-p21 (1:500; Abcam) anti-GRP78 (1:500; Abcam), anti-ZEB1 (1:500; Abcam), anti-MET (1:500; Abcam) and anti- $\beta$-actin (1:500; Abcam) overnight at $4^{\circ} \mathrm{C}$, IRDye ${ }^{\mathrm{TM}}$ 800 conjugated anti-rabbit secondary antibodies (Li-COR, Biosciences, Lincoln, NE, USA) were used for $30 \mathrm{~min}$ at room temperature. The specific proteins were visualized by Odyssey $^{\mathrm{TM}}$ Infrared Imaging System (Neogen Corp., Lincoln, NE, USA).

Immunofluorescence analyses. Immunofluorescence analyses were performed as previously described (31).

Methods of bioinformatics. The analysis of potential microRNA target sites using the commonly used prediction algorithm-miRanda (http://www.targetscan.org//).

Migration and invasion assay. It was performed as previously described (32).

Reverse transcription-polymerase chain reaction and realtime for $m R N A$. It was performed as previously described (33). Primers for HOXD8: forward-5'-TTCCCTGGATGAGAC CACAAGCAGC-3' and reverse-5'-GTCTCTCCGTGAGGG CCAGAGT-3'. Primers for GAPDH: forward-5'-CGGAGTC AACGGATTTGGTCGTAT-3' and reverse-5'-AGCCTTCT CCATGGTGGTGAAGAC-3'.

Statistical analysis. Data are presented as mean \pm SEM. Student's t-test (two-tailed) was used to compare two groups $(\mathrm{P}<0.05$ was considered significant), unless otherwise indicated ( $\chi^{2}$ test).

\section{Results}

Expression of miR-520a-3p is downregulated in NSCLC and SCLC. In an attempt to identify miR-520a-3p expression between the lung cancer tissues and the adjacent normal tissues, we performed real-time PCR in cancer tissues vs. normal tissues. mRNA was isolated from 39 pairs of lung cancer tissues (20 NSCLC tissues and 19 SCLC tissues) and adjacent normal tissues (ANT). We found that miR-520a-3p was significantly decreased in NSCLC and SCLC tissues, compared with their adjacent normal tissues (Fig. 1). It implied that miR-520a-3p could be a tumor suppressive gene in NSCLC and SCLC.

miR-520a-3p inhibits proliferation in NSCLC and SCLC. To investigate whether miR-520a-3p can affect proliferation of NSCLC and SCLC cells, using real-time PCR, we tested whether pre-miR-520a-3p could stably express miR-520a-3p in 

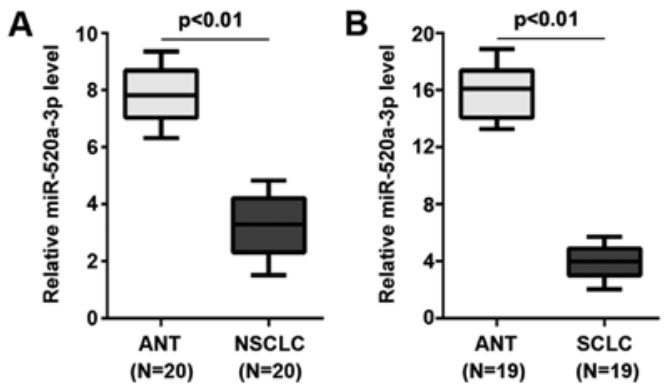

Figure 1. Expression of miR-520a-3p is downregulated in NSCLC and SCLC. (A) Real-time PCR for miR-520a-3p in NSCLC tissues and adjacent normal tissues (ANT). U6 was a loading control. $\mathrm{N}=20$. (B) Real-time PCR for miR-520a-3p in SCLC tissues and adjacent normal tissues (ANT). U6 was a loading control. $\mathrm{N}=19$.

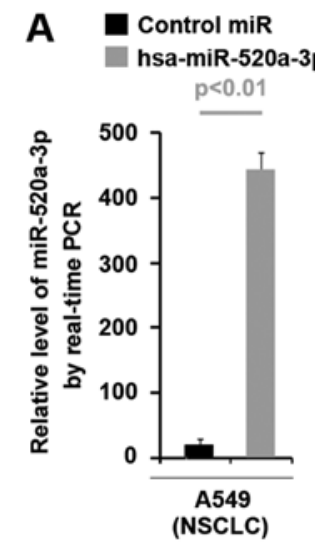

C
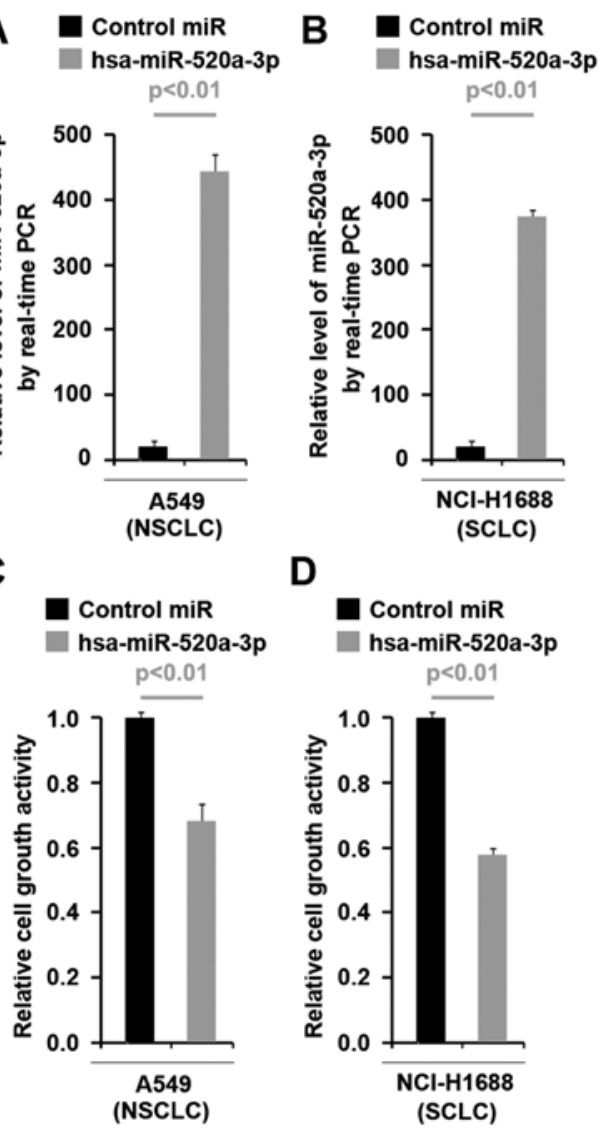

D

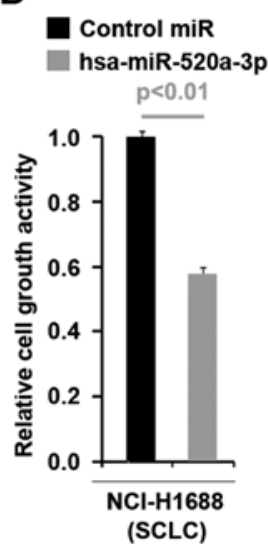

Figure 2. miR-520a-3p inhibits proliferation in NSCLC and SCLC cells. (A) Real-time PCR for miR-520a-3p in NSCLC A549 cells transfected with premiR-520a-3p or control miR (mock). U6 was a loading control. (B) Real-time PCR for miR-520a-3p in SCLC NCI-H1688 cells transfected with pre-miR520a-3p or control miR (mock). U6 was a loading control. (C) MTT assay for NSCLC A549 cells transfected with pre-miR-520a-3p and control miR (mock). (D) MTT assay for SCLC NCI-H1688 cells transfected with premiR-520a-3p and control miR (mock). All experiments were repeated three times, $\mathrm{n}=3$.

A549 cells (NSCLC cells) and NCI-H1688 cells (SCLC cells). The results showed that miR-520a-3p could be significantly increased by pre-miR-520a-3p in the two cell lines (Fig. 2A and B). Next, we performed MTT assay to detect proliferation of A549 cells and NCI-H1688 cells transfected with premiR-520a-3p. The results showed that miR-520a-3p inhibited proliferation in the two cell lines (Fig. 2C and D).
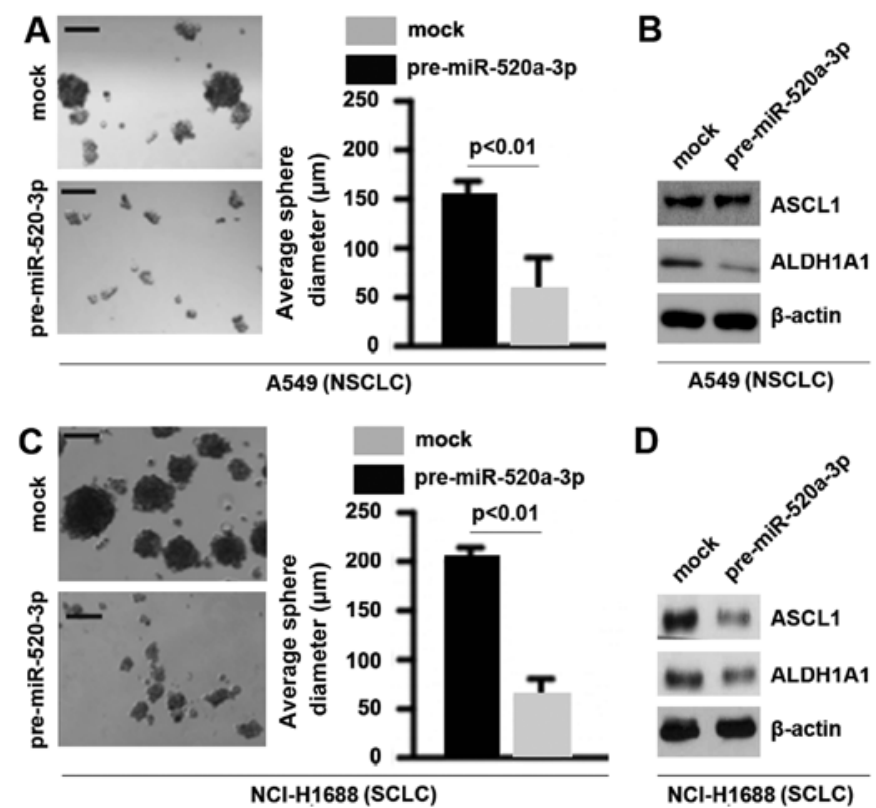

Figure 3. miR-520a-3p inhibits stem cell-like phenotypes in NSCLC and SCLC cells. (A) Sphere growth for NSCLC A549 cells transfected with premiR-520a-3p and control miR (mock). Left panel shows microscopic pictures of sphere growth. Left panel shows graphic presentation of average sphere diameter. (B) Western blot analysis for ASCL1 and ALDH1A1 in A549 cells. A549 cells were transfected with pre-miR-520a-3p or control miR (mock). $\beta$-actin was a loading control. (C) Sphere growth for SCLC NCI-H1688 cells transfected with pre-miR-520a-3p and control miR (mock). Left panel shows microscopic pictures of sphere growth. Left panel shows graphic presentation of average sphere diameter. (D) Western blot analysis for ASCL1 and ALDH1A1 in A549 cells. A549 cells were transfected with pre-miR-520a-3p or control miR (mock). $\beta$-actin was a loading control. All experiments were repeated three times, $n=3$.

miR-520a-3p inhibits stem cell-like phenotypes in NSCLC and SCLC. To determine whether miR-520a-3p could affect formation of CSCs in NSCLC and SCLC, we performed sphere forming assay to assess the capacity of CSC or CSC-like cell self renewal in the present study. We found that formation of spheres was decreased by miR-520a-3p in A549 cells and NCI-H1688 cells (Fig. 3A and C). Achaete-scute complex homolog 1 (ASCL1) is critical for enhanced tumor-initiating capacity in the CD133 ${ }^{\text {high }}$ SCLC sub-population (34). In order to detect whether miR-520a-3p could regulate ASCL1 protein expression in A549 cells and NCI-H1688 cells, we performed western blot analysis to assess ASCL1 protein levels. The results showed that miR-520a-3p cannot regulate ASCL1 protein in A549 cells, but it could significantly suppress the protein in NCI-H1688 cells (Fig. 3B and D). Aldehyde dehydrogenase 1 (ALDH1A1) is a tumor stem cell-associated marker in lung cancer (35). We also performed western blot analysis to detect whether miR-520a-3p could regulate ALDH1A1. The results demonstrated that ALDH1A1 was downregulated in A549 cells and NCI-H1688 cells (Fig. 3B and D).

miR-520a-3p can degrade HOXD8 in NSCLC A549 cells. To search target genes of miR-520a-3p, we commonly used prediction algorithm, TargetScan (http://www.targetscan. org/) to predict its target genes. The algorithm predicted that dozens of target genes could be targeted by miR-520a-3p. We were interested in HOXD8, because we found that contrary 


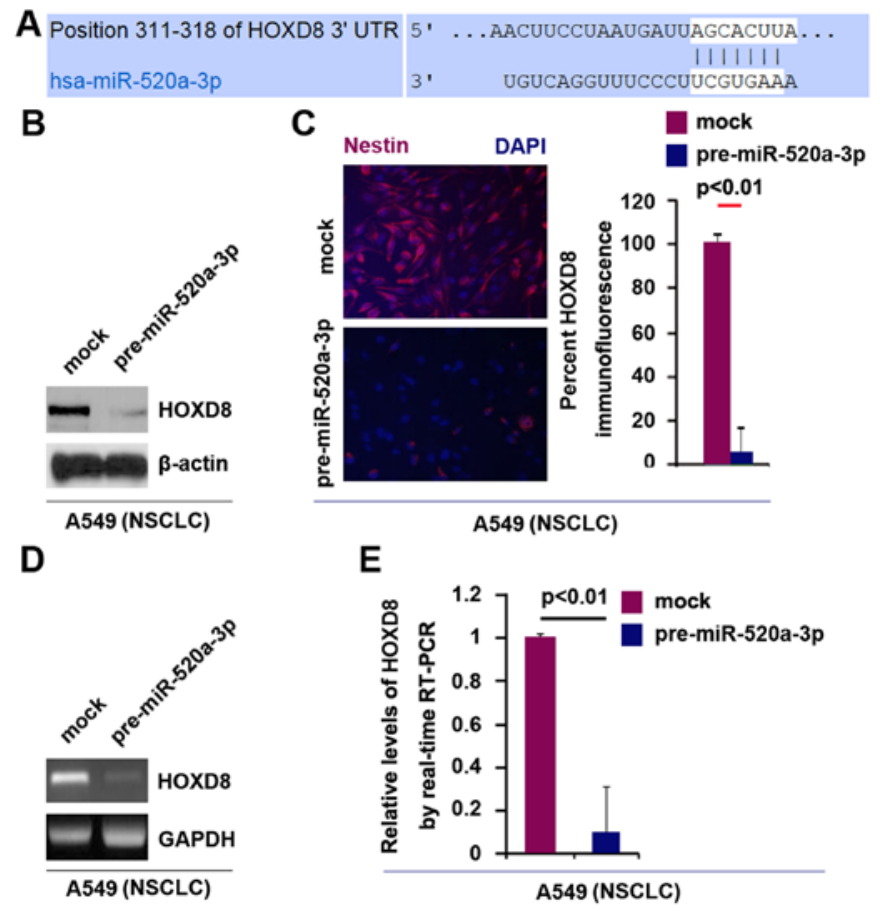

Figure 4. miR-520a-3p can degrade HOXD8 in NSCLC A549 cells. (A) Schematic of predicted miR-520a-3p-binding sites in the 3'UTR of HOXD8 mRNA by TargetScan. (B) Western blot analysis for HOXD8 in A549 cells. A549 cells were transfected with pre-miR-520a-3p or control miR (mock). $\beta$-actin was a loading control. (C) Immunofluorescence analyses for HOXD8 in A549 cells transfected with pre-miR-520a-3p or control miR (mock). (D) RT-PCR for HOXD8 in A549 cells. A549 cells were transfected with pre-miR-520a-3p or control miR (mock). GAPDH was a loading control. (E) Real-time RT-PCR for HOXD8 in A549 cells transfected with pre-miR520a-3p or control miR (mock). U6 was a loading control. All experiments were repeated three times, $n=3$.

to miR-520a-3p, it can promote proliferation and cancer stem cell phenotypes (data shown below). Thus, we reasoned that miR-520a-3p might inhibit proliferation and stem cell-like phenotypes by regulating HOXD8 in NSCLC and SCLC.

Target sites of miR-520a-3p on 3'UTR of HOXD8 are shown in Fig. 4A. In an attempt to identify the role of miR-520a-3p in regulating HOXD8 protein expression in A549 cells, we performed western blot analysis in cells transfected with miR-520a-3p and control miR. The results showed that HOXD8 protein was evidently suppressed in the cells transfected with pre-miR-520a-3p (Fig. 4B). Moreover, we performed immunofluorescence analysis in A549 cells transfected with pre-miR-520a-3p and control miR. Consistent with the results of western blotting, the results of immunofluorescence showed that HOXD8 protein was evidently suppressed in the cells transfected with pre-miR-520a-3p (Fig. 4C).

We next performed RT-PCR and real-time PCR to detect HOXD8 mRNA expression in A549 cells transfected with premiR-520a-3p or control miR. The results of RT-PCR showed that HOXD8 mRNA was significantly downregulated in the cells transfected with pre-miR-520a-3p (Fig. 4D). Consistent with the results of RT-PCR, real-time PCR demonstrated that HOXD8 mRNA was reduced in A549 cells transfected with pre-miR-520a-3p, compared with control miR-transfected groups (Fig. 4E). All the data demonstrated that miR-520a-3p can degrade HOXD8 mRNA expression in NSCLC A549 cells.

\section{A

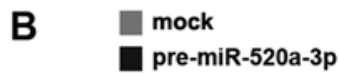

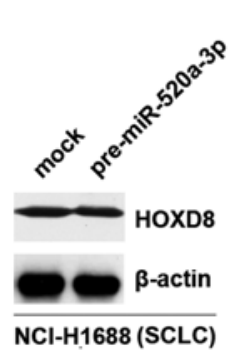

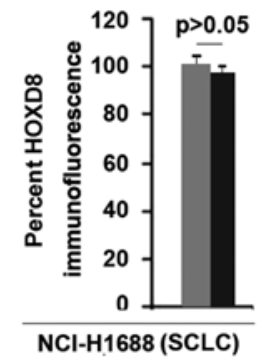

C

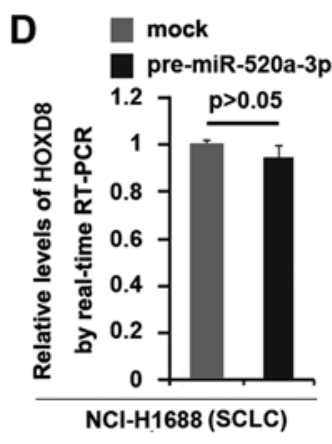

Figure 5. miR-520a-3p cannot suppress HOXD8 expression in SCLC NCI-H1688 cells. (A) Western blot analysis for HOXD8 in NCI-H1688 cells. NCI-H1688 cells were transfected with pre-miR-520a-3p or control miR (mock). $\beta$-actin was a loading control. (B) Immunofluorescence analyses for HOXD8 in NCI-H1688 cells transfected with pre-miR-520a-3p or control miR (mock). (C) RT-PCR for HOXD8 in NCI-H1688 cells. NCI-H1688 cells were transfected with pre-miR-520a-3p or control miR (mock). GAPDH was a loading control. (D) Real-time RT-PCR for miR-520a-3p in NCI-H1688 cells transfected with pre-miR-520a-3p or control miR (mock). U6 was a loading control. All experiments were repeated three times, $n=3$.

miR-520a-3p cannot suppress HOXD8 expression in SCLC NCI-H1688 cells. Having demonstrated that miR-520a-3p can degrade HOXD8 in NSCLC A549 cells, we further studied whether HOXD8 is regulated by miR-520a-3p in SCLC NCI-H1688 cells.

In an attempt to identify the role of miR-520a-3p in regulating HOXD8 protein expression in NCI-H1688 cells, we performed western blot analysis and immunofluorescence analysis in cells transfected with pre-miR-520a-3p and control miR. The results showed that HOXD8 protein was not changed in the cells transfected with pre-miR-520a-3p (Fig. 5A and B). We next performed RT-PCR and real-time PCR to detect HOXD8 mRNA expression in NCI-H1688 cells transfected with pre-miR-520a-3p or control miR. The results of RT-PCR and real-time PCR showed that HOXD8 mRNA was not significantly changed in the cells transfected with premiR-520a-3p (Fig. 5C and D).

HOXD8 is upregulated in cancer tissues and its overexpression can promote proliferation, migration, invasion and cancer stem cell phenotype in NSCLC A549 cells. To identify HOXD8 expression between NSCLC tissues and adjacent normal tissues, we performed western blot analysis in cancer tissues vs. normal tissues. Protein was isolated from 20 NSCLC tissues and 20 adjacent normal tissues. We found that HOXD8 expression was significantly increased in NSCLC tissues, compared with adjacent normal tissues (Fig. 6A). It implied 
A

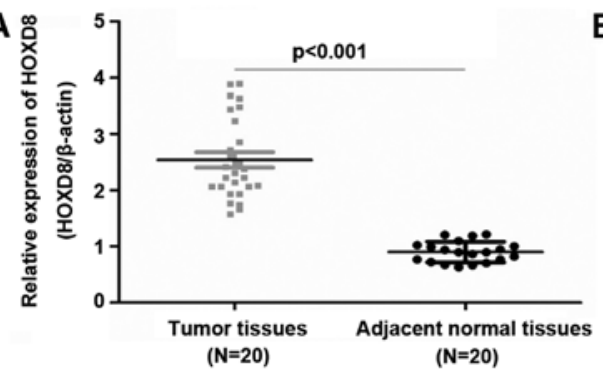

C

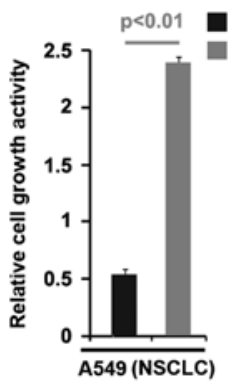

$\mathbf{F}$

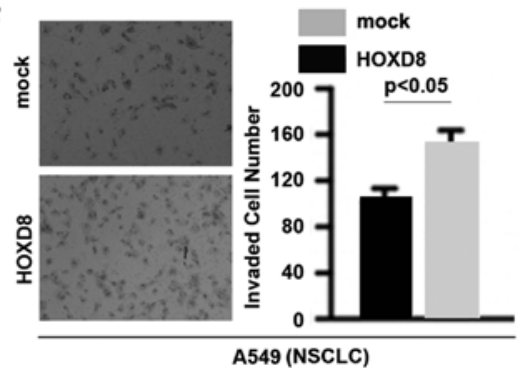

$\mathrm{H}$
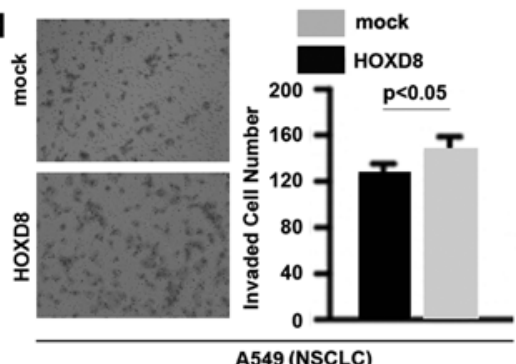

B

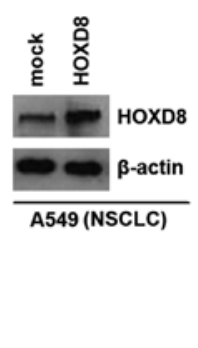

E

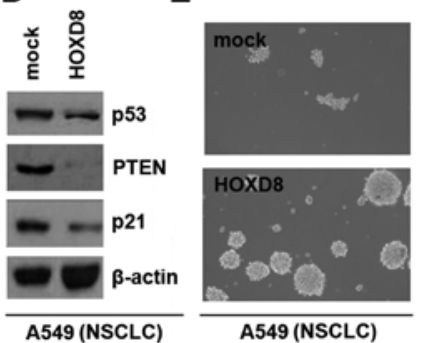

G

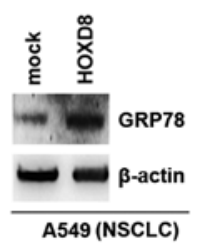

I

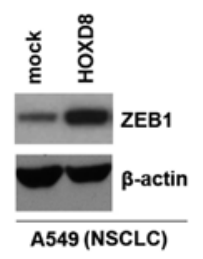

Figure 6. HOXD8 is upregulated in cancer tissues and its overexpression can promote proliferation, migration, invasion and cancer stem cell phenotype in NSCLC A549 cells. (A) Western blot analysis for HOXD8 in NSCLC tissues and adjacent normal tissues. $\beta$-actin was a loading control. $N=20$. (B) Western blot analysis for HOXD8 in A549 cells. The cells were transfected with HOXD8 expressing plasmids or empty vectors (mock). $\beta$-actin was a loading control. (C) MTT assay for A549 cells transfected with HOXD8 expressing plasmids or empty vectors (mock). (D) Western blot analysis for p53, PTEN and p21 in A549 cells. The cells were transfected with HOXD8 expressing plasmids or empty vectors (mock). $\beta$-actin was a loading control. (E) Sphere growth for A549 cells transfected with HOXD8 expressing plasmids or empty vectors (mock). (F) Matrigel invasion assay for A549 cells transfected with HOXD8 expressing plasmids or empty vectors (mock). (G) Western blot analysis for GRP78 in A549 cells. NSCLC A549 cells were transfected with HOXD8 expressing plasmids or empty vectors (mock). $\beta$-actin was a loading control. (H) Transwell migration assay for A549 cells transfected with HOXD8 expressing plasmids or empty vectors (mock). (I) Western blot analysis for ZEB1 in A549 cells. NSCLC A549 cells were transfected with HOXD8 expressing plasmids or empty vectors (mock). $\beta$-actin was a loading control. All experiments were repeated three times, $n=3$.

that HOXD8 could be an oncogene in NSCLC. To investigate whether HOXD8 can affect proliferation ofA549 cells, using
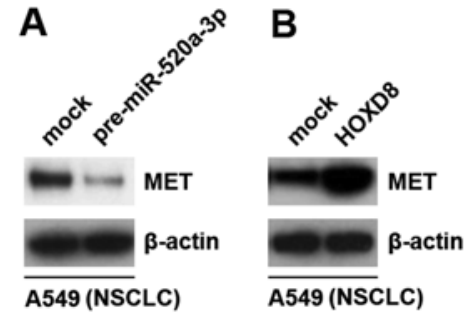

Figure 7. Pre-miR-520a-3p inhibits MET protein expression and HOXD8 induced MET protein expression. (A) Western blot analysis for MET in A549 cells. A549 cells were transfected with pre-miR-520a-3p or control miR (mock). $\beta$-actin was a loading control. (B) Western blot analysis for MET in A549 cells. A549 cells were transfected with HOXD8 or empty vectors (mock). $\beta$-actin was a loading control. All experiments were repeated three times, $n=3$.

western blot, we tested whether HOXD8 expressing plasmids could stably express HOXD8 protein in A549 cells. The results showed that HOXD8 could be significantly increased by HOXD8 expressing plasmids in the cell line (Fig. 6B).

Next, we performed MTT assay to detect proliferation of A549 cells transfected with HOXD8 expressing plasmids and empty vectors. The results showed that HOXD8 promoted proliferation in A549 cells (Fig. 6C). To further confirm that HOXD8 could regulate proliferation, we performed western blot analysis to detect proliferation markers, p53, PTEN and p21 and found that HOXD8 significantly inhibited p53, PTEN and p21 protein expression in A549 cells (Fig. 6D).

To determine whether overexpressing HOXD8 could affect stem cell-like phenotypes in A549 cells, we performed sphere forming assay to assess the capacity of CSC or CSC-like cell self renewal in this study. We found that formation of spheres was increased by HOXD8 in A549 cells (Fig. 6E). In order to detect whether HOXD8 could regulate invasion and migration in A549 cells, we performed invasion and migration assay to assess the role of HOXD8 on A549 cells. The results showed that overexpressing HOXD8 promoted migration and invasion in A549 cells (Fig. 6F and H). Upregulation of glucoseregulated protein 78 (GRP78) expression can promote NSCLC cell invasion (36). To indentify whether GRP78 is regulated by HOXD8, we performed western blot analysis to assess GRP78 protein levels in A549 cells transfected with HOXD8 expressing plasmids and empty vectors. The results showed that GRP78 protein was significantly promoted by HOXD8 in A549 cells (Fig. 6G). Moreover, we found that overexpressing HOXD8 could significantly induce ZEB1 protein expression (Fig. 6I).

Pre-miR-520a-3p inhibits MET protein expression and HOXD8 induces MET protein expression. MET amplification play a pivotal role in gefitinib resistance in lung cancer (30). In order to detect the role of pre-miR-520a-3p and HOXD8 on MET expression, we performed western blot analysis to detect MET protein in A549 cells transfected with pre-miR-520a-3p and control miR. The results demonstrated that miR-520a-3p inhibited MET protein in A549 cells (Fig. 7A). Contrary to miR-520a-3p, we found that overexpressing HOXD8 induced MET protein expression in A549 cells (Fig. 7B). Thus, the results implied that pre-miR-520a-3p downregulation and HOXD8 upregulation might play an important role in gefitinib resistance in NSCLC. 


\section{Discussion}

It was reported that miR-520a-3p is downregulated in NSCLC tissues (29). But there is no report on the expression of miR520a-3p in SCLC. Consistent with the report, we confirmed that miR-520a-3p was downregulated in NSCLC tissues. Moreover, we also demonstrated that miR-520a-3p expression is suppressed in SCLC tissues. The results indicate that miR520a-3p might be a tumor suppressive gene in both NSCLC and SCLC. microRNA-520a-3p can inhibit proliferation, apoptosis and metastasis by targeting MAP3K2 in non-small cell lung cancer (29). Consistent with the report, we showed that overexpressing microRNA-520a-3p suppressed proliferation in NSCLC and SCLC cells.

CSCs assume a central role in both tumorigenesis and metastasis (37). Better understanding of the regulatory mechanisms of CSCs as a fundamental component of the metastatic cascade will lead to novel therapeutic strategies against metastatic cancer. Herein, we demonstrated that overexpressing pre-miR-520a-3p significantly inhibited sphere growth in NSCLC and SCLC cells. ALDH1A1 is a lung tumor stem cell-associated marker (34). We demonstrated that ALDH1A1 protein is inhibited by miR-520a-3p in NSCLC and SCLC cells. Moreover, ASCL1 (achaete-scute complex homolog 1, Mash1), a proneural basic helix-loop-helix (bHLH) transcription factor, was initially identified as a key regulator of early development of mitotically-active precursors for both neurons and oligodendrocytes (38). ASCL1 is highly expressed in classic SCLC and in NSCLC with neuroendocrine (NE) phenotype features (39). ASCL1 regulates tumor-initiating capacity in human small cell lung cancer (34). Our results showed that ASCL1 protein can be suppressed by miR-520a-3p in SCLC cells, but it cannot be suppressed in NSCLC cells highlighting that the different roles of miR-520a-3p in NSCLC and SCLC cells.

In this study, we found that HOXD8 is another target gene of miR-520a-3p in NSCLC, besides MAP3K2. miR-520a-3p could degrade HOXD8 mRNA in NSCLC cells. However, we found that HOXD8 was not suppressed in SCLC cells by miR-520a-3p, implying that miR-520a-3p functions as a tumor suppressive gene by different mechanism in SCLC and NSCLC. For the first time, we showed that HOXD8 protein is upregulated in NSCLC tissues and its overexpression promoted proliferation, cancer stem cell phenotypes, migration and invasion in NSCLC cells.

Gefitinib-sensitive lung cancer cell line can develop resistance to gefitinib as a result of focal amplification of the MET proto-oncogene. Inhibition of MET signaling in lung cells can restore their sensitivity to gefitinib (30). We showed that miR-520a-3p can inhibit MET protein expression and HOXD8 can induce MET protein expression, implying that miR-520a-3p downregulation and HOXD8 upregulation may play an important role in the development of gefitinib resistance.

\section{References}

1. Molina JR, Yang P, Cassivi SD, Schild SE and Adjei AA: Non-small cell lung cancer: Epidemiology, risk factors, treatment, and survivorship. Mayo Clin Proc 83: 584-594, 2008.

2. Yang P: Epidemiology of lung cancer prognosis: Quantity and quality of life. Methods Mol Biol 471: 469-486, 2009.

3. Reya T, Morrison SJ, Clarke MF and Weissman IL: Stem cells, cancer, and cancer stem cells. Nature 414: 105-111, 2001.
4. Dean M, Fojo T and Bates S: Tumour stem cells and drug resistance. Nat Rev Cancer 5: 275-284, 2005.

5. Polyak K, Haviv I and Campbell IG: Co-evolution of tumor cells and their microenvironment. Trends Genet 25: 30-38, 2009.

6. Ponti D, Costa A, Zaffaroni N, Pratesi G, Petrangolini G, Coradini D, Pilotti S, Pierotti MA and Daidone MG: Isolation and in vitro propagation of tumorigenic breast cancer cells with stem/progenitor cell properties. Cancer Res 65: 5506-5511, 2005.

7. Liu G, Yuan X, Zeng Z, Tunici P, Ng H, Abdulkadir IR, Lu L, Irvin D, Black KL and Yu JS: Analysis of gene expression and chemoresistance of $\mathrm{CD}_{133^{+}}$cancer stem cells in glioblastoma. Mol Cancer 5: 67, 2006.

8. Collins AT and Maitland NJ: Prostate cancer stem cells. Eur J Cancer 42: 1213-1218, 2006.

9. Li C, Heidt DG, Dalerba P, Burant CF, Zhang L, Adsay V, Wicha M, Clarke MF and Simeone DM: Identification of pancreatic cancer stem cells. Cancer Res 67: 1030-1037, 2007.

10. Vaiopoulos AG, Kostakis ID, Koutsilieris $M$ and Papavassiliou AG: Colorectal cancer stem cells. Stem Cells 30: 363-371, 2012.

11. Eramo A, Lotti F, Sette G, Pilozzi E, Biffoni M, Di Virgilio A, Conticello C, Ruco L, Peschle C and De Maria R: Identification and expansion of the tumorigenic lung cancer stem cell population. Cell Death Differ 15: 504-514, 2008.

12. Sullivan JP, Minna JD and Shay JW: Evidence for self-renewing lung cancer stem cells and their implications in tumor initiation, progression, and targeted therapy. Cancer Metastasis Rev 29: 61-72, 2010 .

13. Shafee N, Smith CR, Wei S, Kim Y, Mills GB, Hortobagyi GN, Stanbridge EJ and Lee EY: Cancer stem cells contribute to cisplatin resistance in Brca1/p53-mediated mouse mammary tumors. Cancer Res 68: 3243-3250, 2008.

14. To K, Fotovati A, Reipas KM, Law JH, Hu K, Wang J, Astanehe A, Davies AH, Lee L, Stratford AL, et al: Y-box binding protein-1 induces the expression of CD44 and CD49f leading to enhanced self-renewal, mammosphere growth, and drug resistance. Cancer Res 70: 2840-2851, 2010.

15. Calin GA and Croce CM: MicroRNA-cancer connection: The beginning of a new tale. Cancer Res 66: 7390-7394, 2006.

16. Esquela-Kerscher A and Slack FJ: Oncomirs - microRNAs with a role in cancer. Nat Rev Cancer 6: 259-269, 2006.

17. McManus MT: MicroRNAs and cancer. Semin Cancer Biol 13: 253-258, 2003.

18. Farazi TA, Hoell JI, Morozov P and Tuschl T: MicroRNAs in human cancer. Adv Exp Med Biol 774: 1-20, 2013.

19. Reinhart BJ, Slack FJ, Basson M, Pasquinelli AE, Bettinger JC, Rougvie AE, Horvitz HR and Ruvkun G: The 21-nucleotide let-7 RNA regulates developmental timing in Caenorhabditis elegans. Nature 403: 901-906, 2000.

20. Kloosterman WP and Plasterk RH: The diverse functions of microRNAs in animal development and disease. Dev Cell 11: 441-450, 2006.

21. Meng F, Henson R, Wehbe-Janek H, Ghoshal K, Jacob ST and Patel T: MicroRNA-21 regulates expression of the PTEN tumor suppressor gene in human hepatocellular cancer. Gastroenterology 133: 647-658, 2007.

22. Jovanovic $M$ and Hengartner MO: miRNAs and apoptosis: RNAs to die for. Oncogene 25: 6176-6187, 2006.

23. Lu J, Getz G, Miska EA, Alvarez-Saavedra E, Lamb J, Peck D, Sweet-Cordero A, Ebert BL, Mak RH, Ferrando AA, et al: MicroRNA expression profiles classify human cancers. Nature 435: 834-838, 2005.

24. Calin GA and Croce CM: MicroRNA signatures in human cancers. Nat Rev Cancer 6: 857-866, 2006.

25. Croce CM and Calin GA: miRNAs, cancer, and stem cell division. Cell 122: 6-7, 2005.

26. Melton C, Judson RL and Blelloch R: Opposing microRNA families regulate self-renewal in mouse embryonic stem cells. Nature 463: 621-626, 2010.

27. Yu F, Yao H, Zhu P, Zhang X, Pan Q, Gong C, Huang Y, Hu X, Su F, Lieberman J and Song E: let-7 regulates selfrenewal and tumorigenicity of breast cancer cells. Cell 31: 1109-1123, 2007.

28. Shimono Y, Zabala M, Cho RW, Lobo N, Dalerba P, Qian D, Diehn M, Liu H, Panula SP, Chiao E, et al: Downregulation of miRNA-200c links breast cancer stem cells with normal stem cells. Cell 138: 592-603, 2009.

29. Yu J, Tan Q, Deng B, Fang C, Qi D and Wang R: The microRNA520 a-3p inhibits proliferation, apoptosis and metastasis by targeting MAP3K2 in non-small cell lung cancer. Am J Cancer Res 5: 802-811, 2015. 
30. Engelman JA, Zejnullahu K, Mitsudomi T, Song Y, Hyland C, Park JO, Lindeman N, Gale CM, Zhao X, Christensen J, et al: MET amplification leads to gefitinib resistance in lung cancer by activating ERBB3 signaling. Science 316: 1039-1043, 2007.

31. Tang L, Chen F, Pang EJ, Zhang ZQ, Jin BW and Dong WF: MicroRNA-182 inhibits proliferation through targeting oncogenic ANUBL1 in gastric cancer. Oncol Rep 33: 1707-1716, 2015.

32. Dai X, Ge J, Wang X, Qian X, Zhang C and Li X: OCT4 regulates epithelial-mesenchymal transition and its knockdown inhibits colorectal cancer cell migration and invasion. Oncol Rep 29: $155-160,2013$.

33. Zhang HY, Li JH, Li G and Wang SR: Activation of ARK5/ miR-1181/HOXA10 axis promotes epithelial-mesenchymal transition in ovarian cancer. Oncol Rep 34: 1193-1202, 2015.

34. Jiang T, Collins BJ, Jin N, Watkins DN, Brock MV, Matsui W, Nelkin BD and Ball DW: Achaete-scute complex homologue 1 regulates tumor-initiating capacity in human small cell lung cancer. Cancer Res 69: 845-854, 2009.
35. Jiang F, Qiu Q, Khanna A, Todd NW, Deepak J, Xing L, Wang H, Liu Z, Su Y, Stass SA, et al: Aldehyde dehydrogenase 1 is a tumor stem cell-associated marker in lung cancer. Mol Cancer Res 7: 330-338, 2009.

36. Yu T, Guo Z, Fan H, Song J, Liu Y, Gao Z and Wang Q: Cancerassociated fibroblasts promote non-small cell lung cancer cell invasion by upregulation of glucose-regulated protein 78 (GRP78) expression in an integrated bionic microfluidic device. Oncotarget: Mar 21, 2016 (Epub ahead of print). doi: 10.18632/ oncotarget.8232.

37. Li F, Tiede B, Massagué J and Kang Y: Beyond tumorigenesis: Cancer stem cells in metastasis. Cell Res 17: 3-14, 2007.

38. Ball DW: Achaete-scute homolog-1 and Notch in lung neuroendocrine development and cancer. Cancer Lett 204: 159-169, 2004.

39. Sriuranpong V, Borges MW, Strock CL, Nakakura EK, Watkins DN, Blaumueller CM, Nelkin BD and Ball DW: Notch signaling induces rapid degradation of achaete-scute homolog 1 . Mol Cell Biol 22: 3129-3139, 2002. 